\title{
Publisher Correction: Coal-fired power plant closures and retrofits reduce asthma morbidity in the local population
}

Joan A. Casey (D, Jason G. Su, Lucas R. F. Henneman (D), Corwin Zigler, Andreas M. Neophytou, Ralph Catalano (D), Rahul Gondalia @, Yu-Ting Chen, Leanne Kaye, Sarah S. Moyer, Veronica Combs, Grace Simrall, Ted Smith, James Sublett and Meredith A. Barrett

Correction to: Nature Energy https://doi.org/10.1038/s41560-020-0622-9, published online 1 May 2020.

This Policy Brief was originally published incorrectly under an Open Access licence (CC BY 4.0); this has now been amended.

Published online: 14 May 2020

https://doi.org/10.1038/s41560-020-0633-6

๑) Springer Nature Limited 2020 